\title{
Notre chemin vers vos objectifs: stratégie 2017-2020
}

\author{
Jürg Schlup
}

Dr méd., président de la FMH

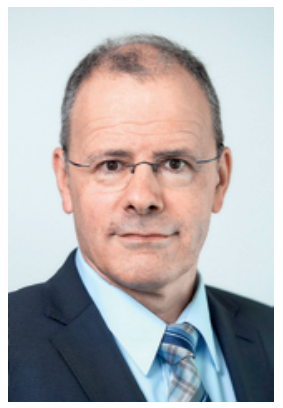

Avec un peu de chance, celui qui prend de bonnes résolutions en janvier appartiendra à la fin de l'année aux quelque 20\% de personnes qui les ont encore en mémoire et qui auront su s'y tenir plus de trois mois. En revanche, il est hors de question qu'une organisation professionnelle forte de 70 organisations médicales s'en remette à de bonnes résolutions ou au hasard. Pour atteindre des objectifs ciblant les intérêts de nos 40000 membres, impliquant la collaboration de nombreuses personnes et acteurs, il faut impérativement élaborer une stratégie fondée, assortie d'objectifs principaux, de sous-objectifs et de mesures pour les atteindre, en attribuant les responsabilités et le contrôle du suivi (controlling).

Quelques-unes des missions qui nous attendent ne pourront être menées à terme sans votre soutien.

C'est dans cet esprit que nous, au Comité central, avons défini la stratégie de la législature en cours. Pour nous assurer que les objectifs retenus reflèteront bien nos enjeux prioritaires, nous avions préalablement demandé aux délégués à la Chambre médicale, à plusieurs experts de la politique de la santé, et aux cadres du Secrétariat général, quels étaient selon eux les défis les plus importants pour la FMH. Sur la base des résultats de ces enquêtes, le Comité central a formulé des objectifs lors de trois ateliers, et mis en avant les mesures concrètes pour les atteindre. Vous en apprendrez davantage dans l'article à la page 4 de ce numéro.

Ces enjeux formulés par le corps médical et nos alliés politiques sont inscrits dans la stratégie, et nous les comprenons comme des missions à poursuivre de manière déterminée. Or quelques-uns ne pourront être menés à terme qu'avec votre soutien; je pense surtout aux priorités phare de notre enquête, à savoir la révision du tarif et le maintien de l'autonomie tarifaire, mais aussi au renforcement de la cohésion intérieure et à notre présentation convergente face au débat public.

Ces priorités sont à relever d'autant plus qu'elles font suite aux vives discussions sur le tarif, qui ont amené à son rejet par 54\% des votants en juin 2016, alors qu'il était clair pour tous qu'il serait impossible de présenter

une proposition meilleure et plus consensuelle dans le court délai imposé par la Confédération. Cet échec n’a pas amélioré notre positionnement politique: sans proposition de tarif, nous sommes les mains vides, alors même que certains parlementaires libéraux réclament un tarif étatique. Nous travaillons donc sous forte pression pour améliorer le tarif proposé, mais nous sommes tributaires du bon vouloir des politiques. En effet, une proposition améliorée de révision tarifaire, soutenue démocratiquement par les organisations médicales et acceptée par les partenaires tarifaires, ne pourra pas être finalisée avant 2018. Pourtant, cette procédure commune est pour nous la seule voie judicieuse, même si cela prend du temps.

Dans cette situation, savoir que le corps médical accorde une grande importance à conserver son autonomie tarifaire et à mener à bien la révision tarifaire, malgré les divergences internes à résoudre, est un préalable nécessaire au succès. Il ne faut cependant pas oublier que le point décisif sera ce que chaque discipline acceptera de concéder. Un appel à l'unité n'inclut-il pas aussi de ne pas tout abandonner dès qu'on doit faire des concessions? Le souhait de cohésion est-il suffisamment fort pour renoncer à des confrontations publiques? L'exigence de solidarité implique-t-elle seulement la solidarité des autres ou englobe-t-elle aussi la nôtre? Si chacun répond oui, alors nous aurons une réelle chance de succès, car autonomie tarifaire et cohésion exigent que toutes les organisations médicales et tous les partenaires tarifaires travaillent à une solution commune, et la défendent, même si la solution choisie ne sera parfaite pour personne, mais c'est un challenge qui en vaut la peine.

Il faut la volonté de tous les acteurs à travailler à une solution qui ne sera pas parfaite mais qui en vaut la peine.

Au travers de notre travail, basé sur la nouvelle stratégie, nous visons le meilleur pour nos membres. A la fin de cette législature, nous connaîtrons tous nos objectifs pour y avoir travaillé bien plus que trois mois. Mais savoir si nous les atteindrons dépendra de la volonté de tous: nous comptons sur vous! 